\title{
Synthesis and Characterization of Lanthanum Nanoparticles by Anethum Graveolens (Dill) Leaf Extract
}

\author{
ROOPA BELURKAR \\ Department of Chemistry Parvatibai Chowgule College of Arts \& Science (Autonomous), \\ Gogol, Margao, Goa, India. \\ *Corresponding author E-mail: belurkar@gmail.com \\ http://dx.doi.org/10.13005/ojc/370527
}

(Received: July 07, 2021; Accepted: September 10, 2021)

ABSTRACT

\begin{abstract}
Anethum Graveolens is a herb used extensively as an additive in chicken feed to help in enhancing the performance, immune reaction and fitness of broiler chickens. The herb consists of various volatile secondary metabolites which are bioactive compounds which helps in their daily food regime. This present work is aimed at synthesizing and characterizing Lanthanumnanoparticles (LaNps) by using Anethum Graveolens (Dill) leaf. LaNps has been synthesized by various methods and characterized by using UV-Vis spectral analysis, Fourier infra-red, X-ray diffraction and SEM analysis.
\end{abstract}

Keywords: Lanthanum Nanoparticles, Synthesis, Anethum Graveolens, Characterization, X-ray diffraction and SEM analysis.

\section{INTRODUCTION}

Nanoparticles have specific and unique modified physical and chemical properties as compared to its macro scaled counterparts ${ }^{1,2}$. Emergence of Nanotechnology has attracted researchers in various fields of technology and industries ${ }^{3}$. Nanoparticles bridge the gap between the bulk fabric, atomic and molecular structures. Nanoparticles finds its use in medicine, pharmacy and dentistry and other fields due to their unique physical and chemical properties such as optical, magnetic, mechanical and conductance ${ }^{4}$. Excessive use of antibiotics has led to microbial resistance, due to which, many researchers have focused on improvement of novel and powerful antimicrobial retainers ${ }^{5,6}$. Several studies have confirmed the antibacterial activity of $\mathrm{Ag}-\mathrm{Nps}$ against a wide range of microorganisms ${ }^{7,8}$. As a result, it may turn out to be a promising compound in development of Novel antimicrobials ${ }^{9}$. Anethum Graveolens is a seed spice and an important aromatic herb used in control of diabetes mellitus, usually known as "Dill seed". It is widely found in Mediterranean region, South West Europe and South West Asia ${ }^{10}$. Anethum Graveolens has many healing effects. It is used as a carminative, digestive and as a tranquilizer. It has also been used in ancient times

This is an Open Access article licensed under a Creative Commons license: Attribution 4.0 International (CC- BY). Published by Oriental Scientific Publishing Company @ 2018 
in treating stomach ailments, colic, hiccups, bad breath, flatulence and hemorrhoids.

Anethum Graveolens has been used extensively in Ayurvedic medicine as a spice and essential oil. It has various volatile components such as Carvone, which is the main component. Along with this, it has a-phellandrene, dill-ether, flavonoids, myristicin, limonene, eugenol, anethole, coumarins, triterpenes, phenolic acids and umbelliferones.

Anethum Graveolens contains distinctive pharmacological effects like anti-cancer, anti-gastric, anti- inflammatory, anti-oxidant, anti-microbial, anti-spasmodic and mucosal protective effects ${ }^{10,11,12}$.

\section{MATERIAL AND METHODS}

The Anethum Graveolens leaves, seeds and stems is washed with sterile distilled water and crushed to fine pieces. This is then filtered through Whatmann filter paper no. 1 . The filter paper is discarded and the filterate is used to extract the essential oil by the process of distillation. The extract obtained from distillation is treated with Lanthanutrate to obtain lanthanum nanoparticle, Lanthanum nitrate also acts as a precursor in the synthesis of Ianthanum nanoparticle. The nanoparticle thus obtained is then dispersed in deionised water to remove any unwanted organic molecules, which gets dissolved in water. The dispersed nanoparticle is then filtered and the filtrate is rejected. The Lanthanum nanoparticle thus obtained is then incubated at $37^{\circ} \mathrm{C}$. The resultant nanoparticle is then used for characterization.

\section{Characterization of Lanthanum nanoparticles}

UV-Vis spectral analysis: The principle of UV-Visible spectroscopy is based on the absorption of ultra violet light or visible light by chemical compounds which results in the production of distinct spectra. When these chemical compounds absorb ultraviolet radiations, it undergoes excitation. This causes it to jump from ground state to an excited state. It is important to know that the difference in the energies of the ground state and the excited state of the electrons is always equal to the amount of ultraviolet radiation or visible radiation absorbed by it, resulting in the production of spectrum. UV-Visible photometer is procured from Systronics. A aliquot of the sample is subjected to the UV-Visible spectrophotometer to obtain a spectrum in the range 200 to $800 \mathrm{~nm}$.

\section{SEM analysis of Lanthanum nanoparticles}

Scanning electron microscopy (SEM) is a test process that scans a sample with an electron beam to produce a magnified image for analysis. The signals generated during this analysis produces two dimensional image and produces information about the sample. It mainly describes the texture, chemical composition and orientation of the samples under investigation. The SEM used for analysis is Jeol version jsm6390 lv SEM system. The film used for analysis were prepared on a carbon coated copper grid by using a small quantity of the Lanthanum nanoparticle.

\section{FTIR Spectrophotometer}

The nanoparticle is dried and powdered and is subjected to FTIR (Schimadzu Japan) spectrophotometer for analysis. The analysis indicated the formation of Lanthanide nanoparticles.

\section{X-ray diffraction analysis}

The X-ray diffraction analysis were obtained from Bruker, D-8 Venture, Germany, which operated at a voltage of $40 \mathrm{KV}$.

\section{RESULTS AND DISCUSSIONS}

UV-Vis Spectra Analysis: The density of Lanthanum nanoparticles was confirmed by obtaining the UV-Vis spectrum of the Lanthanide nanoparticle solution with a high absorption at $212.2 \mathrm{~nm}$; and the concentration details reveal that the particles were scattered by monochromatic light.The electronic spectrum of Lanthanum 
nanoparticles is shown in Fig. 1. The absorption band $212.2 \mathrm{~nm}$ can be assigned to the conversion of the intra-ligand aromatic $\pi \rightarrow \pi^{\star}$ aromatic ring $\mathrm{n}-\pi^{\star}$ nitrate. There is a change in the field of view due to $d^{0}$ electron configuration in Lanthanum (III). UV-Visible spectroscopy is a simple and quick way to confirm the formation of Lanthanide nanoparticles.

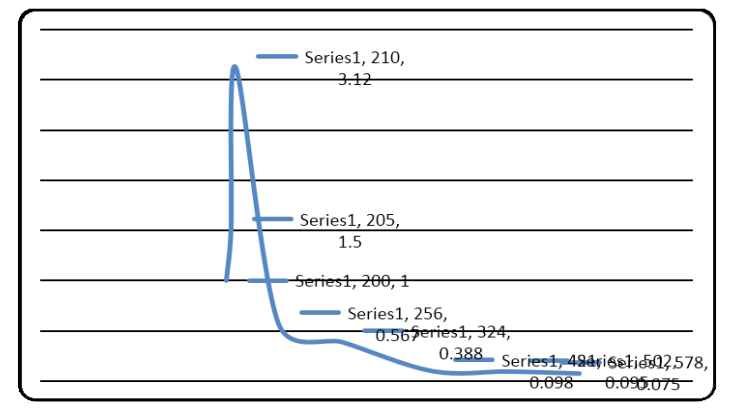

Fig.1. UV-spectra of LaNPs

\section{FTIR Analysis}

FTIR is used to identify the biomolecules present in the Dill plant extract, such as peptides, which helps in reducing LaNPs and is also used as a capping reagent for the reduced LaNPs ${ }^{13,14,15,16}$. The typical FTIR spectrum for mixed LaNPs and Dill leaf extraction is shown in (Fig. 2). Strong absorption peaks are identified at $3309 \mathrm{~cm}^{-1}$ from the extension of the $\mathrm{NH}$-band (amino groups) or $\mathrm{OH}$ band (hydroxyl group) due to the phenols of Dill plant extract. The absorption peak of $2916.37 \mathrm{~cm}^{-1}$ can explain the vibration of $\mathrm{CH}$ in alkanes. A strong band observed at $1599 \mathrm{~cm}^{-1}$, is due to $-\mathrm{NH}_{2}$ groups of amino acids. The peaks at 1443.83 and $1243 \mathrm{~cm}^{-1}$ explains $\mathrm{CH}$ vibrations due to ketonic group

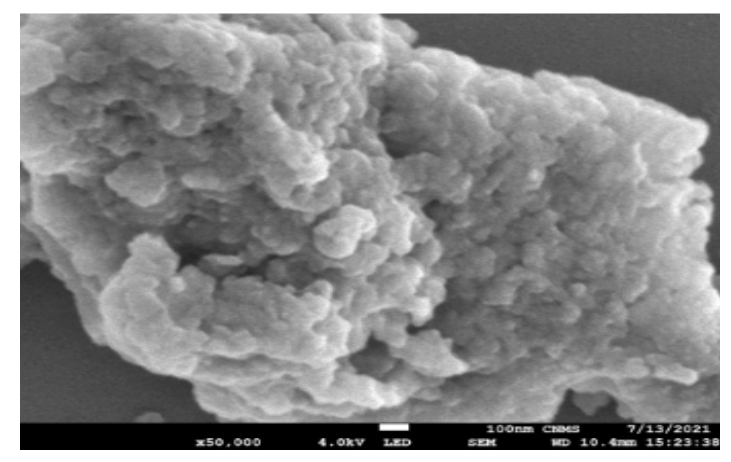

Fig. 3. and $\mathrm{CO}$ group respectively. A strong band identified at $1035 \mathrm{~cm}^{-1}$ is due to PO-stretching. Also LaNPs showed strong absorption peaks at $686,1531,1675,2348$, and 3753 $\mathrm{cm}^{-1}$; A band with a strength of $686 \mathrm{~cm}^{-1}$ is due to $-\mathrm{CH}$ - without vibration bending the plane with ethylene systems replaced by $-\mathrm{CH}=\mathrm{CH}$ - (cis). A strong band at $2348 \mathrm{~cm}^{-1}$ is due to a silver-linked $\mathrm{CN}$-bond from natural sources ${ }^{17}$ indicating the presence of NPs in the sample. A height of $1675 \mathrm{~cm}^{-1}$ represents a $\mathrm{C}=\mathrm{O}$ extension of the proteins or peptides formed as a capping agent found in dill leaf extraction. Finally, the presence of a solid peak at $3753 \mathrm{~cm}^{-1}$ is due to amines and is an evidence of the presence of peptides in LaNPs.

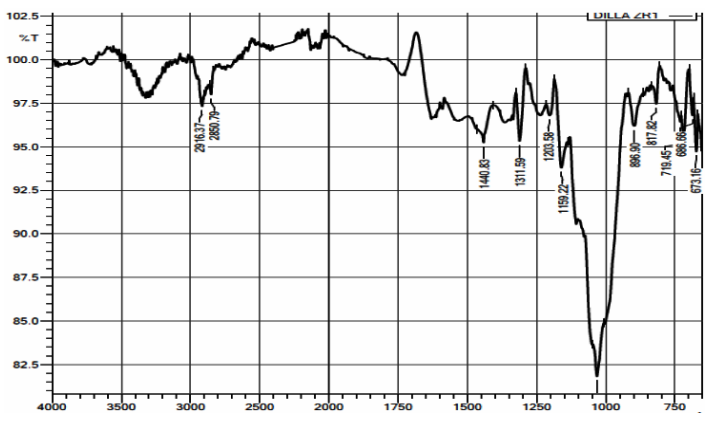

Fig. 2. IR Spectra of LaNPs

\section{SEM analysis}

SEM analysis confirmed that the Lanthanum nanoparticles produced in this study were nano size. They are round in shape and have a polidispersing distance of about $10.4 \mathrm{~nm}$ (Fig. 3-6). Nanoparticles did not mix after ten days. This suggests that Dill leaf extract can act as a reducing and stabilizing agent.

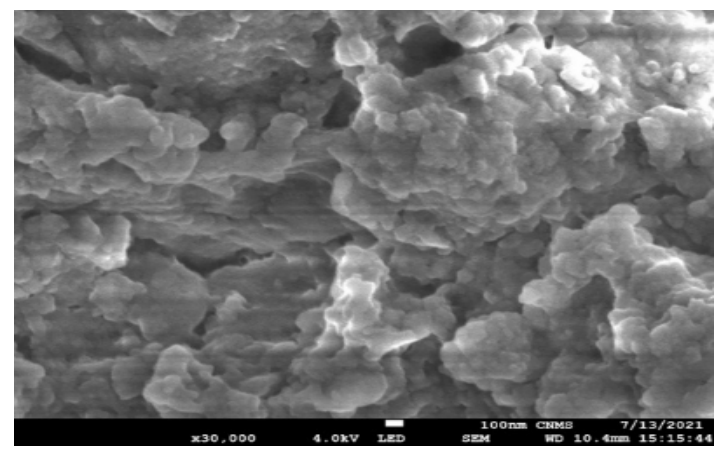

Fig. 4. 


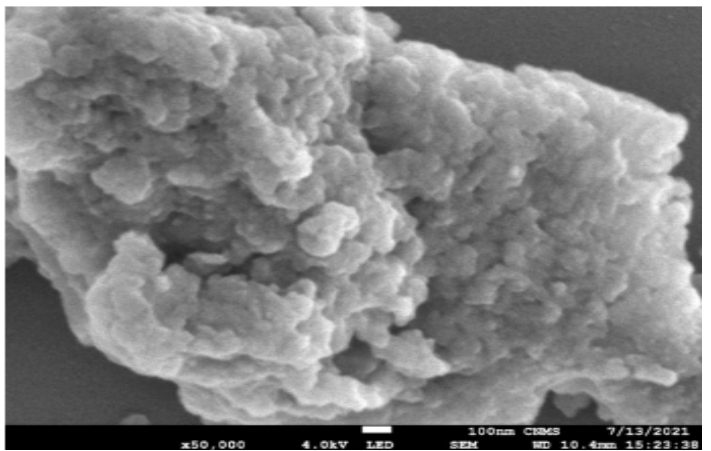

Fig. 5.

\section{XRD analysis of LaNPs}

The crystalline nature of the Lanthanum nanoparticles was clearly analyzed using XRD patterns. Separated peaks of Lanthanum nanoparticles appear at 28.29, 41.85, 48.1 and 72.0 (Fig. 7). Non-allocated sets of 111, 200, 220, 311 aircraft were also stored compared to JCPDS data.

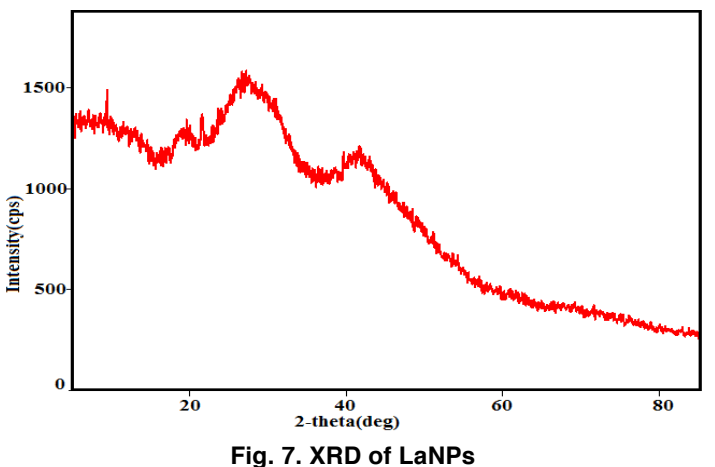

CONCLUSION

In the field of nano-biotechnology,

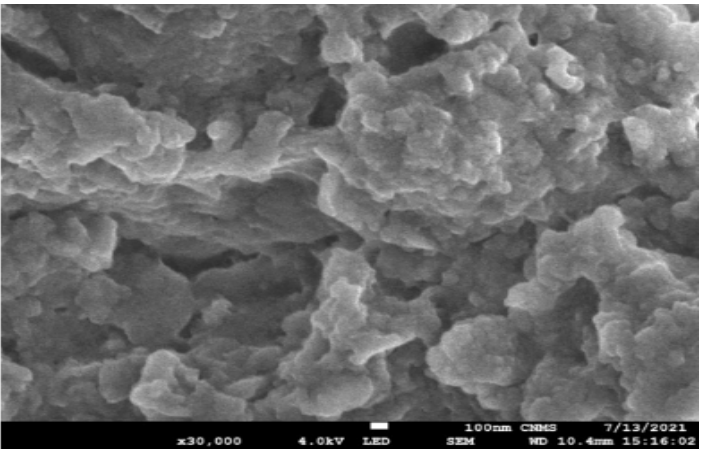

Fig. 6.

phytosynthesis of nanoparticles has been used to produce nutritious, inexpensive materials, containing stable nanoparticles which is of great importance to its widespread application in the field of electronics, medicine and agriculture. In the current context, nanotechnology helps in promoting progress in all aspects of life, which is why, the phytosynthetic method of nanoparticles synthesis will emerge as one of the safest and most advanced among the conventional methods.

\section{ACKNOWLEDGEMENT}

The author is thankful to the Principal and Management of Parvatibai Chowgule College of Arts \& Science (Autonomus), Margao, Goa, India.

\section{Conflict of interest}

The author declare that we have no conflict of interest.

\section{REFERENCES}

1. Raveendran, P.; J. Fu.; S. L. Wallen.; J. Am. Chem. Soc., 2003, 125, 13940.

2. Li, L.; Hu J.; Alivistos, A. P.; Nano lett., 2001, $1,349$.

3. Sattarahmady, N.; Movahedpour A.; Heli H, Hatam G. R.;. Sensors and Actuators B: Chemical., 2016, 1, 235:723-31.

4. Shabaninejad, Z.; Yousefi, F.; Movahedpour, A.; Ghasemi, Y.; Dokanehiifard, S.; Rezaei S, Anal Biochem., 2019, 15(581), 113349.

5. Thombre, R. S.; Shinde, V.; Thaiparambil, E.; Zende, S.; Mehta, S.; Frontiers in microbiology., 2016, 7, 1424.

6. Sana, S. S.; Dogiparthi, L. K.; Material letters., 2018, 226, 47-5.

7. Kim. S. H.; Lee, H. S.; Ryu, D. S.; Choi, S. J.; Lee, D. S.; Korean J. Microbiol. Biotechnol., 2011, 39(1), 77-85.

8. Sondi, I.; Salopek-Sondi, B.; Journal of Colloid and Interface Technology., 2004, 275(1), 177- 82.

9. Siddiqi, K. S.; Husen, A.; Journal of nanobiotechnology., 2018, 16(1), 14. 
10. Nidhi, M.;. J. Veterinary international., 2013 6(8), 502-507.

11. Majid, M.; Laleh, P.; Alireza, O.; Yasir, K. B.; Mohammad, A. J.; J. Pharmaceutical Sciences., 2014, 20(45), 40-45.

12. Fatemeh, H.; Mehernoosh, Z.; Fatemeh, B.; Kambiz, A. A.; Golnaz, A.; Magazine of Isfahan medical faculty., 2015, 32(320), 2015: 2473-2483.

15. Mosmann, T.; J. Immunol methods., 1983,
65, 55-63.

16. Sahib, A. S.; Mohammad, I. H.; Al-Gareeb, A. I.; J. Spatula D D., 2012, 2, 153-8.

17. Arora, D. S.; Kaur, G.; J. Nat Med., 2007, 61, 313-7.

18. Kulikova, O.; Maltsev, D.; Ilyina, A.V.; Burdina, V. P.; Yamskova, V. P,: Yamskov, I. A.; J. Prikl Biokhim Microbiol., 2015, 51, 362-6.

19. Miller, F. A.; Wilkins, C. H.; Analytical Chemistry., 1952, 24, 1253-94. 